\title{
A fault diagnosis based reconfigurable longitudinal control system for managing loss of air data sensors for a civil aircraft ${ }^{\star}$
}

\author{
A. Varga D. Ossmann H.-D. Joos \\ Institute of System Dynamics and Control, DLR Oberpfaffenhofen, \\ D-82234 Wessling, Germany. \\ (e-mail: \{andreas.varga, daniel.ossmann, dieter.joos \}@dlr.de)
}

\begin{abstract}
An integrated fault diagnosis based fault tolerant longitudinal control system architecture is proposed for civil aircraft which can accommodate partial or total losses of angle of attack and/or calibrated airspeed sensors. A triplex sensor redundancy is assumed for the normal operation of the aircraft using a gain scheduled longitudinal normal control law. The fault isolation functionality is provided by a bank of 6 fault detection filters, which individually monitor each of the 6 sensors using robust low order LPV residual generators. In the case of losses of up to 5 sensors, a fault estimation technique based on LPV estimators can be employed to reconstruct the missing sensor information necessary for gain scheduling. In the worst case of a total failure of all 6 sensors, a robust constant longitudinal control law is employed which ensures a basic longitudinal control performance. The proposed control architecture fulfils the basic requirements formulated in the Benchmark Problem in the RECONFIGURE project.
\end{abstract}

Keywords: Flight control system, fault detection and isolation, sensor faults, fault estimation, fault tolerant control.

\section{INTRODUCTION}

The normal longitudinal control law for civil aircraft provides high performance operation over the full range of operating points in presence of various parametric and operational uncertainties. Typically, a gain scheduling based controller is employed, where the main scheduling parameters are the calibrated air speed $\left(V_{c}\right)$ and the Mach number $(M)$. Occasionally, available estimated inertial information as the weight $(w)$ and center of gravity position $\left(X_{c g}\right)$ can be also used to adapt the controller gains. Additionally, on Airbus machines, the normal longitudinal law also provides pitch axis and angle of attack (AoA) $(\alpha)$ protections.

One of the recent trends in designing flight control systems is to try to preserve the longitudinal normal law performance even in the presence of faults [Puyou and Ezerzere, 2012] (see also [Oudin et al., 2012] for an alternative approach in the lateral axis). Thus, by avoiding switching to the alternate or direct control laws, with limited or even lost protections, it is aimed to keep the nominal control performance even in the case of failures, without increasing the pilot's workload.

In this paper we address this problematic in the case of air data sensor faults, and specifically faults in the $V_{c}$ and AoA sensors. These two measurements play important roles both in implementing the robust gain scheduling based longitudinal control algorithm (e.g., $V_{c}$ ), but also for the AoA protection. A triplex sensor redundancy is nowadays for both $V_{c}$ and AoA measurements a standard

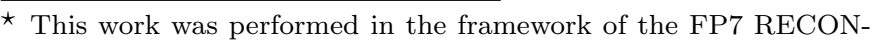
FIGURE Project: Grant agreement AAT-2012-RTD-2314544.
}

requirement to accommodate single sensor faults in each of these sensors [Goupil, 2011].

The cases of simultaneous failures of two $V_{c}$ or two AoA sensors require the use of more involved fault diagnosis techniques to isolate the faulty sensors. An individual monitoring of AoA sensors has been proposed by Ossmann and Varga [2011] for the detection and isolation of any of the AoA sensor fault, by using all available measurements (including $V_{c}$ ). Thus two or even three simultaneous AoA sensor faults can be isolated using low order linear parameter varying (LPV) residual generator filters. In this paper we employ a similar approach for monitoring the six individual sensors for $V_{c}$ and AoA, with the difference that, the designed LPV residual generators for monitoring the AoA sensor faults do not use measurements from the $V_{c}$ sensors, while the designed LPV residual generators for monitoring the $V_{c}$ sensor faults do not use measurements from the AoA sensors. This allows a robust isolation of all six potential sensor faults and thus provides valuable information for possible fault estimation.

As long as at least one sensor in each category is healthy, the provided measurements can be used to preserve the normal control law and associated protection. This is in principle possible, even in the case, when only one sensor measurement is valid. For example, in the case of failure of all AoA sensors, the guaranteed healthy measurement of $V_{c}$ can be used to build a robust fault estimator of the AoA sensor faults, which thus can be used to build a virtual AoA sensor to serve for preserving the AoA protection. Similarly, a healthy AoA sensor allows to reconstruct the $V_{c}$ fault, and thus to build a virtual $V_{c}$ sensor which can be 
used for gain scheduling purposes. These fault estimation aspects are also investigated in this paper and represent the basis of a new approach for a fault accommodating normal longitudinal control law. Alternative methods to simultaneously estimate $V_{c}$ and AoA have been recently proposed in [Seren et al., 2013, Hardier et al., 2013].

The monitoring of simultaneous sensor faults $V_{c}$ and AoA is one of the fault detection and isolation (FDI) benchmark scenarios formulated in the FP7 Project RECONFIGURE (Reconfiguration of Control in Flight for Integral Global Upset Recovery). A complementary benchmark scenario addresses the complete loss of all $V_{c}$ and AoA sensors. In such a case, maintaining the longitudinal normal law, with possibly reduced handling performance (but still providing altitude hold and level change capabilities), and keeping the aircraft in a safe region (but without active protection) appear as reasonable goals for the design of a robust backup control law. In this paper we also address the design of a robust backup longitudinal control law based on a $C^{*}$-control algorithm, which fulfills these requirements, without using any gain scheduling.

\section{A RECONFIGURABLE LONGITUDINAL CONTROL ARCHITECTURE}

In this section we describe the proposed reconfigurable longitudinal flight control architecture shown in Fig. 1 to manage the partial or total loss of air data sensors for $V_{c}$ and AoA for a civil aircraft. In what follows, we describe succinctly the functionality of the main blocks.

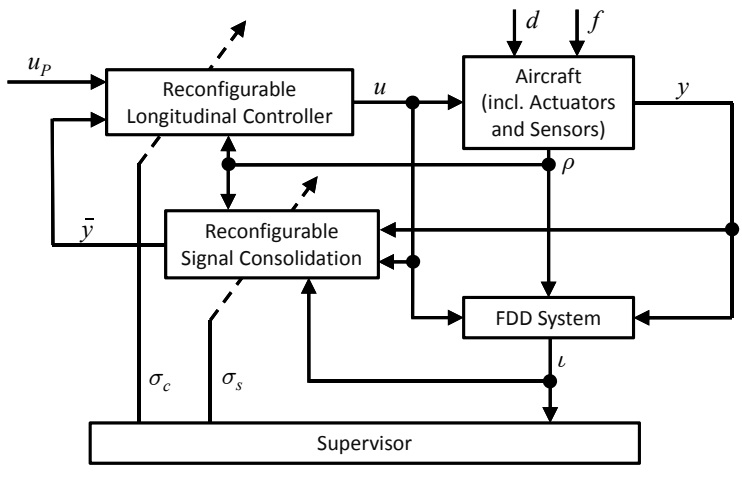

Fig. 1. FDD-based FTC architecture (longitudinal axis)

The open-loop aircraft is equipped with suitable actuators, to control the primary surface deflections via the input signal $u$, and sensors, to produce the measurement signal $y$. The wind components $d$ and sensor faults $f$ are exogenous inputs also acting on the aircraft. In the longitudinal axis, the components of the control input vector $u$ are the demanded elevator and stabilizer deflections, while $y$ includes the main measurements as the air data sensors for $V_{c}$ and $\alpha$ (both with triplex redundancy) as well as measurements used by the longitudinal control algorithm or the fault monitoring algorithms, as the vertical load factor $N_{z}$, pitch rate $q$, height $h$, pitch angle, etc. Several flight parameters are collected into the vector $\rho$ and can serve for specific gain scheduling purposes. Typical components of $\rho$ are $V_{c}, M, w$ or $X_{c g}$.

The Reconfigurable Longitudinal Controller block consists internally of two interchangeable blocks, as shown in
Fig. 2. Both the nominal controller and the backup controller process the pilot input $u_{P}$ (usually a $N_{z}$ demmand) and a set of consolidated measurements $\bar{y}$ and generate the actuator command $u$. While the nominal controller is usually a gain-scheduled controller using the scheduling parameters in $\rho$, the backup controller does not use gainscheduled gains or the scheduling is performed with a restricted set of parameters (e.g., only $w$ and $X_{c g}$ ). The switching from the nominal to the backup controller is commanded by setting the switching signal from $\sigma_{c}=0$ to $\sigma_{c}=1$ and only occurs if the full air data sensor information on $V_{c}$ and $\alpha$ are lost.

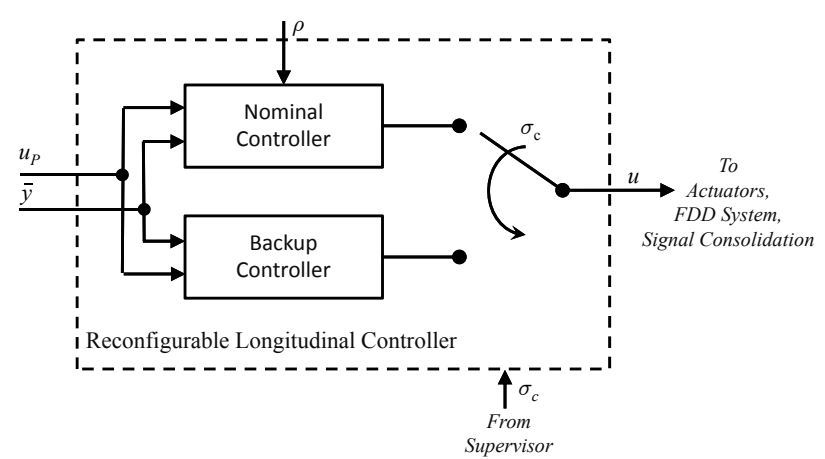

Fig. 2. Reconfigurable longitudinal controller.

The Fault Detection and Diagnosis (FDD) System block is detailed in Fig. 3. Its main functionality is to provide the necessary fault isolation information to allow the supervisor to issue various reconfiguration commands. The isolation information vector $\iota$, generated by two FDI blocks, consists of two sub-vectors $\iota_{\alpha}$ and $\iota_{V_{c}}$ with components set to either 0 (fault) or 1 (no fault), respectively. The FDI system for each category of signals (i.e., AoA and $V_{c}$ ) consists of a bank of three detectors, which monitor each measurement individually. An important requirement for the monitoring of AoA signals, is that no measurements from $V_{c}$ are employed, and similarly the monitoring of $V_{c}$ must not use AoA measurements. A further requirement is the robustness of the fault detection in the presence of operational point variations and parametric uncertainties. For this purpose, fault detection filters with gain scheduling (via the signal $\rho$ ) are used.

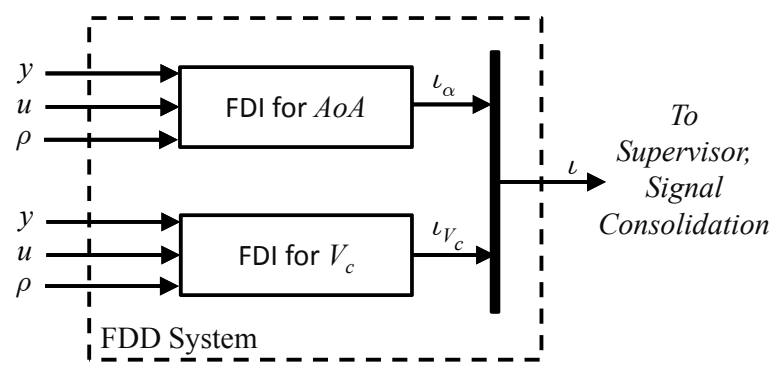

Fig. 3. Fault detection and diagnosis system.

The Reconfigurable Signal Consolidation block is detailed in Fig. 4. Its main functionality is to generate consolidated measurements of the AoA and $V_{c}$ signals, based on the available fault isolation information determined by the FDD System. The voting based consolidation is employed if at least one sensor in a category is healthy. For example, 
in the case of the AoA sensor with three measurements $\alpha_{i}$, $i=1,2,3$, this is simply

$$
\bar{\alpha}=\frac{\sum_{i=1}^{3} \iota_{\alpha_{i}} \alpha_{i}}{\sum_{i=1}^{3} \iota_{\alpha_{i}}}
$$

where $\iota_{\alpha_{i}}$ is the $i$-th component of $\iota_{\alpha}$ (see Fig. 3). A similar formula is used to compute $\bar{V}_{c}$.

If all sensors in a category are faulty, then a virtual sensor can be used, which computes an estimation of the missing sensory information. The main challenge of building virtual sensors is the requirement for its robustness, i.e., to compute plausible estimation in presence of variations of operating point and various parametric uncertainties. This is why, a gain scheduling approach for the observer design is necessary. As an example, a solution for a virtual AoA sensor using healthy $V_{c}$ measurements for gain scheduling is described later in this paper.

The decision on which type of signal consolidation to be used is taken by supervisor, via the switching signals $\sigma_{s, \alpha}$ and $\sigma_{s, V_{c}}$, which are set to 0 or 1 , respectively, in accordance with the available current faulty or healthy sensor information. The resulting consolidated measurement vector $\bar{y}$ includes the updated sensory information $\bar{\alpha}$ and $\bar{V}_{c}$, as well as the rest of measurements grouped in a vector $y_{1}$.

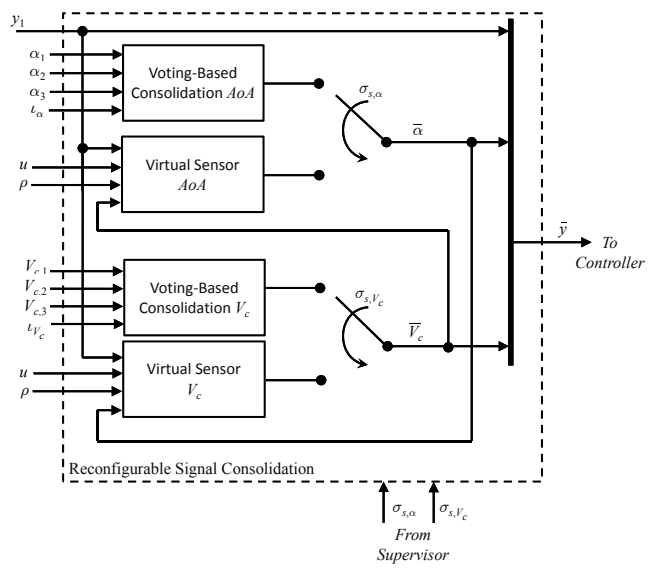

Fig. 4. Reconfigurable signal consolidation.

The Supervisor block processes the fault isolation information provided in the vector $\iota$ and makes suitable reconfiguration decisions by generating the reconfiguration commands $\sigma_{s}$ and $\sigma_{c}$. The reconfiguration of the signal consolidation block occurs with $\sigma_{s}$ in the case when the full sensory information is lost for a category of signals (AoA or $V_{c}$ ). Then, according to the available healthy measurements, a virtual sensor can be employed to provide estimations of missing measurements. In the case of a total failure of all AoA and $V_{c}$ sensors, a controller reconfiguration command $\sigma_{c}$ must be issued. For the implementation of the supervisor, a simple event based logic can be used to generate the reconfiguration commands.

\section{SYNTHESIS METHODS FOR FAULT ISOLATION AND ESTIMATION FILTERS}

In this section we describe synthesis techniques which can be used to solve the fault isolation problems for air data sensors, and the fault estimation problems for designing virtual sensors, in the case when all sensors in a category are lost. The methods we describe are based primarily on linear synthesis techniques, where the robustness aspect is addressed by using multiple linearized longitudinal aircraft models.

\subsection{Synthesis of robust LPV residual generators}

Consider a set of $N$ linearized models with additive sensor faults, where the $i$-th system is described by

$$
\begin{aligned}
& \dot{x}^{(i)}(t)=A^{(i)} x^{(i)}(t)+B_{u}^{(i)} u(t)+B_{d}^{(i)} d(t)+B_{f}^{(i)} f(t) \\
& y^{(i)}(t)=C^{(i)} x^{(i)}(t)+D_{u}^{(i)} u(t)+D_{d}^{(i)} d(t)+D_{f}^{(i)} f(t)
\end{aligned}
$$

and where $x^{(i)}(t)$ and $y^{(i)}(t)$ are, respectively, the $n$ dimensional state vector and the $p$-dimensional output vector of the $i$-th system, and $u(t), d(t)$ and $f(t)$ are the $m_{u^{-}}, m_{d^{-}}$, and $m_{f}$-dimensional control, disturbance and fault input vectors, respectively. In the case of only sensor faults, $B_{f}^{(i)}=0$ and the matrix $D_{f}^{(i)}$ is formed from columns of the $p$-th order identity matrix $I_{p}$. We denote by $G_{u}^{(i)}(s), G_{d}^{(i)}(s)$, and $G_{f}^{(i)}(s)$, the corresponding transfer-function matrices (TFMs) from the corresponding plant inputs $u(t), d(t)$ and $f(t)$ to the outputs $y^{(i)}(t)$, respectively, given for $\xi=u, d$, and $f$ by

$$
G_{\xi}^{(i)}(s)=C^{(i)}\left(s I-A^{(i)}\right)^{-1} B_{\xi}^{(i)}+D_{\xi}^{(i)}
$$

The multiple model representation (2) corresponds in general to $N$ distinct flight conditions characterized by $N$ distinct values of a certain parameter vector $\rho$, i.e., $\rho \in \Pi:=\left\{\rho^{(1)}, \ldots, \rho^{(N)}\right\}$. Thus, the multiple-model (2) can be interpreted as the instantiation for $\rho=\rho^{(i)}$ of the linear parameter varying (LPV) model

$$
\begin{aligned}
& \dot{x}(t)=A(\rho) x(t)+B_{u}(\rho) u(t)+B_{d}(\rho) d(t)+B_{f}(\rho) f(t) \\
& y(t)=C(\rho) x(t)+D_{u}(\rho) u(t)+D_{d}(\rho) d(t)+D_{f}(\rho) f(t)
\end{aligned}
$$

where $A^{(i)}=A\left(\rho^{(i)}\right), B_{u}^{(i)}=B_{u}\left(\rho^{(i)}\right)$, etc. The corresponding TFMs for constant $\rho$ from $u(t), d(t)$ and $f(t)$ to $y(t)$ are denoted by $G_{u}(s, \rho), G_{d}(s, \rho)$, and $G_{f}(s, \rho)$, respectively.

In what follows, we assume that $\rho$ has two components: $\rho_{1} \in \Pi_{1}$, which is not measurable, and $\rho_{2} \in \Pi_{2}$, which is measurable, and $\Pi=\Pi_{1} \times \Pi_{2}$. The synthesis problem to be formulated bellow attempts to solve basically a robust fault detection problem with respect to $\rho_{1}$, while taking advantage of the availability of $\rho_{2}$ by attempting to achieve robustness using an LPV gain scheduling approach.

To solve robust fault detection problems we use a LPV residual generator (or fault detection filter) in the statespace form

$$
\begin{aligned}
& \dot{x}_{Q}(t)=A_{Q}\left(\rho_{2}\right) x_{Q}(t)+B_{Q}\left(\rho_{2}\right)\left[\begin{array}{l}
y(t) \\
u(t)
\end{array}\right] \\
& r(t)=C_{Q}\left(\rho_{2}\right) x_{Q}(t)+D_{Q}\left(\rho_{2}\right)\left[\begin{array}{l}
y(t) \\
u(t)
\end{array}\right]
\end{aligned}
$$

where the matrices $A_{Q}\left(\rho_{2}\right), B_{Q}\left(\rho_{2}\right), C_{Q}\left(\rho_{2}\right)$, and $D_{Q}\left(\rho_{2}\right)$ depend only on the gain scheduling variable $\rho_{2}$. The inputoutput form of the filter (4) is

$$
\mathbf{r}(s)=Q\left(s, \rho_{2}\right)\left[\begin{array}{l}
\mathbf{y}(s) \\
\mathbf{u}(s)
\end{array}\right],
$$


where the bolded quantities denote Laplace transformed variables and $Q\left(s, \rho_{2}\right)$ is the corresponding TFM

$$
Q\left(s, \rho_{2}\right)=C_{Q}\left(\rho_{2}\right)\left(s I-A_{Q}\left(\rho_{2}\right)\right)^{-1} B_{Q}\left(\rho_{2}\right)+D_{Q}\left(\rho_{2}\right)
$$

The residual signal $r(t)$ in (4) generally depends via the system outputs $y(t)$ of all system inputs $u(t), d(t)$ and $f(t)$. By expressing $\mathbf{y}(s)$ in terms of TFMs, the resulting residual generation system (5) becomes

$$
\mathbf{r}(s)=R_{u}(s, \rho) \mathbf{u}(s)+R_{d}(s, \rho) \mathbf{d}(s)+R_{f}(s, \rho) \mathbf{f}(s)
$$

where with

$$
\left[R_{u}(s, \rho)\left|R_{d}(s, \rho)\right| R_{f}(s, \rho)\right]:=Q\left(s, \rho_{2}\right) G_{e}(s, \rho)
$$

$$
G_{e}(s, \rho):=\left[\begin{array}{c|c|c}
G_{u}(s, \rho) & G_{d}(s, \rho) & D_{f} \\
I_{m_{u}} & 0 & 0
\end{array}\right]
$$

For a successfully designed filter $Q\left(s, \rho_{2}\right)$, the corresponding residual generation system is proper with respect to variable $s$, robustly stable and achieves specific fault detection requirements (e.g., exact or approximate decoupling of control and disturbance inputs from the residuals).

We can now formulate the following Robust Fault Detection and Isolation Problem (RFDIP): For the multiplemodel description (2) and a given $m_{f} \times m_{f}$ diagonal reference model $M_{r}(s)$, determine a stable linear residual generator in the LPV-form (4) such that for all $\rho \in \Pi$

(i) $\quad\left\|\left[R_{u}(s, \rho) R_{d}(s, \rho)\right]\right\| \approx 0$

(ii) $\quad\left\|R_{f}(s, \rho)-M_{r}(s)\right\| \approx 0$.

Here $\|\cdot\|$ denotes an appropriate TFM-norm.

Condition $(i)$ requires that the control and disturbance inputs are almost decoupled from the residual signal, while (ii) imposes via the diagonal structure of $M_{r}(s)$ that the $i$-th residual component is only influenced by the $i$-fault component and almost decoupled of the rest of faults.

This model matching formulation can be employed to formulate the fault estimation problem, which requires the complete reconstruction of the fault signal from the residual. Thus, the Robust Fault Estimation Problem (RFEP) is a RFDIP with the special choice $M_{r}(s)=I_{m_{f}}$ of the reference model.

Weaker requirements in the formulations of the RFDIP and RFEP are possible to facilitate the solution of these problems. For example, to solve a RFDIP one can only require that $R_{f}(s, \rho)$ has (approximately) the same zero/nonzero structure as $M_{r}(s)$, which can be chosen simply the identity matrix, or even a (non-diagonal) rectangular structure matrix (with only $0 / 1$ elements). A weaker version of the RFEP is to impose only a diagonal structure for $M_{r}(s)$, with an arbitrarily selectable dynamics, such that $r(t)$ follows arbitrarily fast $f(t)$.

\subsection{Nullspace based solution of RFDIP}

For the solution of the RFDIP we employ the nullspace method based synthesis approach of Varga [2011b] to design a bank of $m_{f}$ LPV fault detection filters with scalar outputs, where the $k$-th filter approximately decouples all control and disturbance inputs, as well as $m_{f}-1$ faults and is only sensitive to the $k$-th fault. For this purpose, we use a multiple-model based synthesis framework, where we aim to design for the $i$-th model in (2) (e.g., corresponding to the parameter value $\left.\rho^{(i)}\right), m_{f}$ scalar fault detection filters
$Q_{k}^{(i)}(s), k=1, \ldots, m_{f}$, which exactly decouples all control and disturbance inputs, as well as $m_{f}-1$ faults and is only sensitive to the $k$-th fault. Using the parametrization result of [Varga, 2011a], it is possible to determine $Q_{k}^{(i)}(s)$ with a state-space realization of least order having the form

$$
\begin{aligned}
& \dot{x}_{Q_{k}}^{(i)}(t)=A_{Q_{k}} x_{Q_{k}}^{(i)}(t)+B_{Q_{k}}^{(i)}\left[\begin{array}{l}
y(t) \\
u(t)
\end{array}\right] \\
& r_{k}^{(i)}(t)=C_{Q_{k}} x_{Q_{k}}^{(i)}(t)+D_{Q_{k}}^{(i)}\left[\begin{array}{l}
y(t) \\
u(t)
\end{array}\right]
\end{aligned}
$$

where the matrices $A_{Q_{k}}$ and $C_{Q_{k}}$ are common to all $N$ models. If this goal can be achieved, then the $N$ values of matrices $B_{Q_{k}}^{(i)}$ and $D_{Q_{k}}^{(i)}$ can be employed to obtain parametric representations for $B_{Q_{k}}\left(\rho_{2}\right)$ and $D_{Q_{k}}\left(\rho_{2}\right)$ (e.g., by employing robust parameter fitting techniques, see [Varga, 2011a] for suitable methods). The $k$-th LPV filter is obtained in the form

$$
\begin{aligned}
& \dot{x}_{Q_{k}}(t)=A_{Q_{k}} x_{Q_{k}}(t)+B_{Q_{k}}\left(\rho_{2}\right)\left[\begin{array}{l}
y(t) \\
u(t)
\end{array}\right] \\
& r_{k}(t)=C_{Q_{k}} x_{Q_{k}}(t)+D_{Q_{k}}\left(\rho_{2}\right)\left[\begin{array}{l}
y(t) \\
u(t)
\end{array}\right]
\end{aligned}
$$

while the resulting overall LPV filter in (4) is obtained by stacking the $k$ resulting LPV filters and has the state-space matrices

$$
\begin{gathered}
A_{Q}\left(\rho_{2}\right)=\left[\begin{array}{ccc}
A_{Q_{1}} & & \\
& \ddots & \\
& & A_{Q_{m_{f}}}
\end{array}\right], \quad B_{Q}\left(\rho_{2}\right)=\left[\begin{array}{c}
B_{Q_{1}}\left(\rho_{2}\right) \\
\vdots \\
B_{Q_{m_{f}}}\left(\rho_{2}\right)
\end{array}\right] \\
C_{Q}\left(\rho_{2}\right)=\left[\begin{array}{c}
C_{Q_{1}} \\
\vdots \\
C_{Q_{m_{f}}}
\end{array}\right], \quad D_{Q}\left(\rho_{2}\right)=\left[\begin{array}{c}
D_{Q_{1}}\left(\rho_{2}\right) \\
\vdots \\
D_{Q_{m_{f}}}\left(\rho_{2}\right)
\end{array}\right]
\end{gathered}
$$

\subsection{Optimization based solution of RFEP}

To solve the RFEP (thus also the RFDIP), we employ an optimization based technique to solve $m_{f}$ associated model matching problems by assuming the form (9) for the $k$-th filter. In this case, the matrices $A_{Q_{k}}$ and $C_{Q_{k}}$ can be set to nominal synthesis values, while for $B_{Q_{k}}\left(\rho_{2}\right):=B_{Q_{k}}\left(\theta, \rho_{2}\right)$ and $D_{Q_{k}}\left(\rho_{2}\right):=D_{Q_{k}}\left(\theta, \rho_{2}\right)$ we can assume a certain type of parametric dependence of a parameter vector $\theta$ (e.g., affine). Then, a convex optimization approach can be formulated to minimize simultaneously the norms in $(i)$ and $(i i)$ for the $k$-th detector, using the following multiobjective minimization based worst-case formulation

$$
\min _{\theta} \max _{\rho \in \Pi}\left\{\left\|\left[R_{u}(s, \rho) R_{d}(s, \rho)\right]\right\|,\left\|R_{f}^{k}(s, \rho)-e_{k}\right\|\right\}
$$

where $R_{f}^{k}(s, \rho)$ and $e_{k}$ are the $k$-th rows of matrix $R_{f}(s, \rho)$ and of the identity matrix $I_{m_{f}}$, respectively. Suitable computational tools based on the method developed in [Apkarian and Noll, 2006] to solve this non-smooth optimization problem are available in the Robust Control Toolbox of MATLAB (function systune in the $2013 \mathrm{~b}$ version).

\section{ROBUST CONTROLLER DESIGN METHODS}

In this section we describe optimization-based techniques suitable to solve robust control synthesis problems using fixed structure controller. For the design of a backup robust longitudinal controller to be used in the case of 
simultaneous failure of all air data sensors, we use an optimisation based multi-objective tuning approach of the controller parameters as described in [Joos, 1999] using the associated software tools [Joos et al., 2002]. Important features of this methodology are that various kinds of design objectives can be taken into account in their most natural form via appropriately formulated mathematical criteria (e.g., initial response, overshoot, settling time), while the robustness requirements can be addressed using various types of synthesis models (e.g., multiple linearized models, LPV models, nonlinear parametric simulation models, etc.). The main appeal of the multi-objective optimisation, as a computer aided design technique, is its ability to handle several (potentially) conflicting design goals simultaneously, and finding the best compromising solution using the computed Pareto-optimal solutions. This technique can also serve for robustness assessment purposes, by finding "worst-case" parameter combinations to decide whether a design is robust or not.

Let $c_{i}(\theta, \rho), i \in \mathcal{I}$ (e.g., $\mathcal{I}=\left\{1, \ldots, N_{c}\right\}$ ) be a set of criteria depending on the tuning parameters $\theta \in \Theta$ and uncertain parameters $\rho \in \Pi$, where $\Theta$ defines the admissible tuning parameter space and $\Pi$ is the set of uncertain parameter values. For example, the previously defined discrete-set $\Pi=\left\{\rho^{(1)}, \ldots, \rho^{(N)}\right\}$ naturally allows a multiple-model based problem formulation. To find satisfactory compromising values for the tuning variable $\theta$ in presence of uncertainties in $\rho$, we can solve a scalar weighted min-max optimisation problem of the form

$$
\begin{aligned}
& \min _{\theta \in \Theta} \max _{i \in \mathcal{I}_{s}, \rho \in \Pi}\left\{\frac{c_{i}(\theta, \rho)}{d_{i}}\right\}, \\
& \text { subject to } \max _{\rho \in \Pi} c_{i}(\theta, \rho) \leq d_{i} \text { for } i \in \mathcal{I} \backslash \mathcal{I}_{s}
\end{aligned}
$$

where the weighting factors $d_{i}>0$ for the $i$-th criterion can be interpreted either as a demand (or soft constraint) for $i \in \mathcal{I}_{s}$ or a hard constraint for $i \in \mathcal{I} \backslash \mathcal{I}_{s}$.

The above min-max multi-criteria optimisation problem can be reformulated as a smooth nonlinear programming problem (NLP) with inequality and simple bounds constraints. This NLP-problem can then be solved by using available solvers as those presented in [Joos et al., 2002]. Besides efficient gradient-based solvers (well-suited primarily for smooth problems), less efficient, but usually more robust gradient-free direct-search based solvers are available to address problems with non-smooth or noisy criteria. Such kind of criteria often occur when engineering design specification are directly translated into optimisation criteria or when truncation errors manifest in the criteria (e.g., from numerical simulation, approximations, etc.). To overcome the problem of local minima to some extent, global strategies based on statistical or deterministic search methods and evolutionary computing can be alternatively or additionally used.

Fixed structure constant longitudinal controllers have been tuned in [Puyou and Ezerzere, 2012] by solving nonsmooth $\mathcal{H}_{\infty}$ optimization problems in frequency domain using the methods developed in [Apkarian and Noll, 2006] for multiple-models. However, handling stability or time response constraints (as overshoot, settling-time, etc.) requires special tricks (e.g., definition of suitable reference models or weighting functions). Also, these methods can- not be used when the underlying plant is nonlinear or is a black box model (e.g., a precompiled Simulink block).

\section{SETUP OF THE RECONFIGURABLE LONGITUDINAL CONTROL SYSTEM}

A set of $N=214$ linear models of the form (2) describing the open-loop linearized aircraft longitudinal dynamics have been used for synthesis purposes. The models have been generated in different nominal trim conditions, which are defined by values of the aircraft weight $w$, center of gravity position $X_{c g}$, velocity $V_{c}$ and altitude $h$. All models have order $n=5$ with the states: AoA $\alpha$, pitch rate $q$, ground speed $V_{g}$, pitch angle $\theta$ and aircraft altitude $h$. Beside the five states, the output vector $y$ of dimension $p=8$ includes the vertical velocity $V_{z}$ and the normal accelerations of the aircraft, $N_{x}$ and $N_{z}$, in the $x$ and $z$ directions, respectively. The elements of the input vector $u$ of dimension $m_{u}=5$ are the horizontal stabilizer position $\delta_{h s}$ and the four elevator deflections (left inner, left outer, right inner, right outer) $\delta_{e, i}$, with $i=1, \ldots, 4$. As measurable parameters the weight $w$ and the center of gravity $X_{c g}$ of the aircraft are available and can be used for controller or fault detection filter gain scheduling. A wind input along $x$-axis is included together with additive sensor fault inputs in all $\alpha$ and $V_{g}$ measurements (recall the existing triplex redundancy). Note that these faults represent simultaneously the additive faults in the $\alpha$ and $V_{c}$ sensors (only in no wind condition). In all designs we used $m_{d}=1$ and $m_{f}=2$.

\subsection{Setup of robust FDI filters}

For the design of the residual generators, a nullspace-based approach described in Section 3 has been applied using the 214 linear open-loop models. For each of the three $\alpha_{g}$ and three $V_{g}$ measurements, a residual filter with a scalar output has been determined. The nullspace method reveals that for all 214 models, zero order filters (i.e., pure gains with only $D_{Q_{1}}^{(i)}$ and $D_{Q_{2}}^{(i)}$ nonzero in (8)) can be used to detect and isolate the sensor faults. For example, to isolate AoA sensor faults, the corresponding residual signal $r_{1}^{(i)}$ is

$$
r_{1}^{(i)}=\alpha^{(i)}-\theta^{(i)}+V_{z}^{(i)} / V_{c}^{(i)}
$$

and expresses the static relationship among the flight-path angle $\gamma \approx V_{z} / V_{c}$, the AoA $\alpha$ and the pitch angle $\theta$.

For the synthesis of the FDI systems in Fig. 3, a diagonal structure matrix can be automatically achieved for each pair of measurements $\left(\alpha, V_{g}\right)$, by simply excluding the speed measurements when detecting faults in the AoA sensors, and similarly, by excluding the AoA measurements when detecting $V_{c}$ sensor faults. When monitoring AoA sensor faults, $\rho_{2}=\left(w, X_{c g}, h\right)$ has been used as scheduling variable, while $\rho_{1}=V_{c}$ as uncertain non-measurable parameter for which robustness of isolation must be guaranteed. When monitoring $V_{c}$ faults, we employed additionally $V_{c}$ as scheduling variable, taking into account that false alarms can be avoided as long as $V_{c}$ is a healthy measurement, while faulty $V_{c}$ will additionally amplify the model mismatch, and thus help in the detection of faults. The validation of this gain scheduling concept will require further analysis of the FDD system in a closed-loop aircraft control configuration. 
As example, we consider the design of a robust fault isolation filter for an AoA sensor, which must be robust against any velocity change. A hybrid gain scheduling has been adopted, where a continuous LPV model based gain scheduling is strived for $w$ and $X_{c g}$, while a discrete gain scheduling is employed for $h$ using 5 distinct values of the altitude. As the filter parameters are nearly constant for a fixed altitude, but vary strongly with any altitude change, this approach appears to be the most suited to ensure the robustness of fault isolation.

A robust FDI filter is designed as follows. Assume there are $N_{2}$ distinct combinations of values of $\rho_{2}=\left(w, X_{c g}, h\right)$ in $\Pi$, and to each value $\rho_{2}^{(j)}, j=1, \ldots, N_{2}$ there are $K_{j}$ values of $V_{c}$. First, we determine for each value $\rho_{2}^{(j)}$ a constant residual filter which provides robust isolation performance for $K_{j}$ values of $V_{c}$. This can be done by using the optimization-based approach described in Section 3.3, where in the optimization problem (10), $\theta$ is the gain $D_{Q_{1}}^{(j)}$ and worst case search is performed over the $K_{j}$ values of $V_{c}$. To speed up the computation, the search can be initialized using the gain computed with the nullspace based approach for one of the intervening models. In a second step, for each distinct value $h^{(i)}$ of $h$, a fitting technique is applied to obtain a parametric form of the corresponding gain $D_{Q_{1}}^{(i)}\left(\tilde{\rho}_{2}\right)$ of the filter, where $\tilde{\rho}_{2}=$ $\left(w, X_{c g}\right)$. Satisfactory performance can be achieved by enforcing an affine dependence of the parameters in $\tilde{\rho}_{2}$. A similar approach can be employed to determine $D_{Q_{2}}^{(i)}\left(\tilde{\rho}_{2}\right)$.

In Fig. 5 simulation results are presented, which illustrate the robustness of the FDI of AoA faults using the designed gain scheduling based residual generator. In the first diagram, the residual response to a typical dynamic load factor command maneuver is depicted, where a deviation of $\delta N_{z}=0.4 \mathrm{~g}$ from the trim position over $15 \mathrm{~s}$ is commanded by the pilot. The results show that for all variations in the velocity, weight and altitude, the residual remains relatively small. In the second diagram the response to a typical wind turbulence with a standard deviation of $\sigma=2$ knots is depicted. Satisfactory decoupling is maintained for all points in the flight envelope. The third diagram shows the response to a slow AoA sensor drift with a freezing at $4 \mathrm{deg}$. In this case a suitable threshold can be easily selected, enabling the detection of the fault while avoiding false alarms.

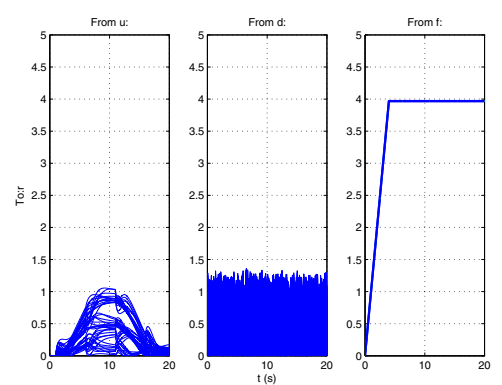

Fig. 5. Residual signal responses to load factor command, wind disturbance and sensor fault

The FDD system in Fig. 3 consists practically of 6 independently working FDI filters, which deliver the isolation information used by all reconfigurable blocks of the FTC system in Fig. 1. No reconfiguration is performed as long as at least one AoA sensor and at least one $V_{c}$ sensor are healthy. Thus, two simultaneous AoA sensor faults and/or two simultaneous $V_{c}$ faults can be easily accommodated, just employing the available isolation information in vectors $\iota_{\alpha}$ and $\iota_{V_{c}}$ to perform the signal consolidation according to (1). These situations can not be managed by pure (voting) signal based consolidation schemes, and completely wrong results are obtained in the (highly improbable) case when two sensor faults are equal.

Figure 6 shows the simulation results of such a double AoA sensor failure at time $t_{f}$ during the typical load factor maneuver. The simple signal based signal consolidation cannot isolate the faults, leading to a erroneous consolidated AoA signal (red plot). In the case of model based FDI, the two faulty sensors are correctly isolated after a short interval (about 1s) at time $t_{d}$ and switched off. The faulty signal consolidation can be thus fully corrected after $t_{d}$, by using the remaining healthy sensor (green plot).

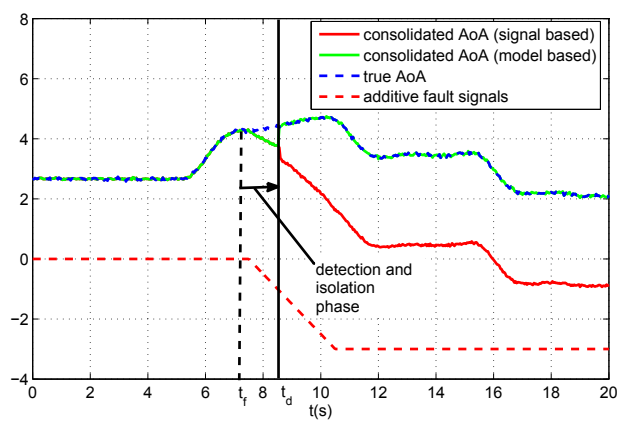

Fig. 6. Signal consolidation for simultaneous AoA faults

\subsection{Setup of virtual sensors}

The fault isolation information provided by the FDD system in Fig. 3 (via $\iota_{\alpha}$ and $\iota_{V_{c}}$ ) serves to fully assess the status of all sensors (i.e., faulty or healthy) and allows to appropriately reconfigure the signal consolidation block in Fig. 4 or the controller block (i.e., by switching to a backup controller in the case of total failure). In the case when all sensors of a category are faulty, but at least one sensor is healthy in the other category, then, in principle, a virtual sensor can be devised to replace the missing sensory information. For example, in the case of failures of all AoA sensors, a virtual measurement $\hat{\alpha}$ can be computed as

$$
\hat{\alpha}=\alpha-\hat{f}_{\alpha}
$$

where $\hat{f}_{\alpha}$ is an estimation of the AoA fault signal $f_{\alpha}$ in the $\alpha$ measurement employing any healthy measurement of $V_{c}$. Similarly it is possible to reconstruct $V_{c}$ if at least one AoA sensor is healthy. The estimated values of the AoA can serve to maintain the AoA protection in the normal control law, while the estimated value of $V_{c}$ can serve for gain scheduling purposes.

The solution of the fault estimation problem for any of the 214 models using the nullspace method revealed that a zero order fault estimator can be locally employed. Therefore, we used a fault estimator of the parametric form

$$
\hat{f}_{\alpha}=D_{\alpha}\left(\rho_{2}\right)\left[\begin{array}{l}
y(t) \\
u(t)
\end{array}\right]
$$


where $\rho_{2}=\left(V_{c}, w, X_{c g}, h\right)$ serves as scheduling variable. An optimization-based tuning procedure, completely similar to that employed in the previous section, has be used to determine $D_{\alpha}\left(\rho_{2}\right)$ by assuming an affine dependence of the scheduling parameters.

In Fig. 7 simulation results are presented for a threefold AoA sensor failure occurring at $t_{f}$ during a typical load factor maneuver. After the detection of total failure at $t_{d}$, the supervisor switches from the sensor signal consolidation scheme (1) to the virtual sensor (12). The results show that the absolute estimation error (red plot) stays in the limit of $1 \mathrm{deg}$, providing a satisfactory estimate of the AoA and enabling to further keep the AoA protection law.

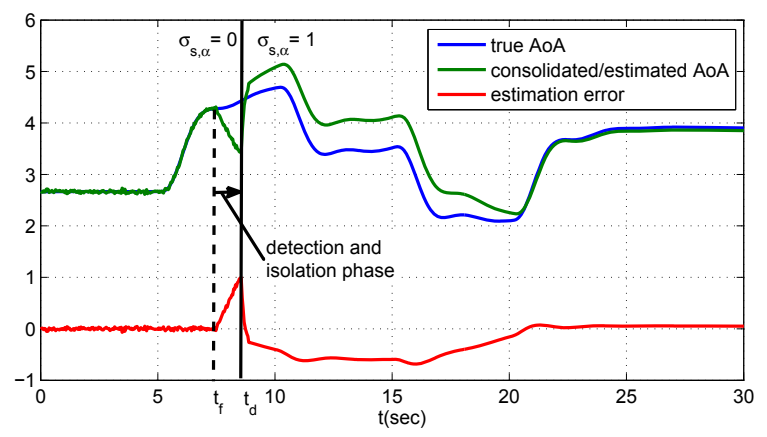

Fig. 7. Switching to an estimated AoA value

\subsection{Tuning of a robust backup controller}

We consider the tuning of the inner control loop of a longitudinal flight control system with the goal of achieving a satisfactory short-term longitudinal dynamics of the aircraft. Several approaches to design longitudinal flight controllers are surveyed in [Puyou and Ezerzere, 2012], where a tuning method of a constant longitudinal controller with fixed structure is proposed using non-smooth optimization techniques. In this section we describe an alternative tuning approach employing the multiple-model framework which is based on general purpose solvers for multi-objective optimization problems available in the MOPS tool [Joos et al., 2002]. Two advantages with respect to the more specialized non-smooth optimization based tuning techniques were instrumental for our choice: the possibility to address arbitrary type of criteria (including time responses based criteria) and to employ arbitrary parameter dependent synthesis models (e.g., nonlinear aircraft simulation model).

We chose as backup inner loop controller a $C^{*}$ control law of a special form which computes the equivalent elevator command $u_{e}(t)$ employing $q_{f}$, the filtered pitch rate $q$, the handling quality related signal $C^{*}(t)=N_{z}(t)-1+V_{m} q(t)$ (built using the vertical load factor $N_{z}(t)$ and velocity factor $\left.V_{m}\right)$, and the equivalent pilot performance reference demand $C_{r e f}^{*}(t)$ built from the commanded load factor $N_{z_{c}}(t)$. The signals $q_{f}(t)$ and $C_{r e f}^{*}(t)$ are generated using first-order lead-lag filters as

$$
\mathbf{q}_{f}(s)=k_{q} \frac{T_{1}^{q} s+1}{T_{2}^{q} s+1} \mathbf{q}(s), \quad \mathbf{C}_{r e f}^{*}(s)=k_{c} \frac{T_{1}^{c} s+1}{T_{2}^{c} s+1} \mathbf{N}_{z_{c}}(s)
$$

The employed $C^{*}$ control law has the form

$$
\begin{aligned}
u_{e}(t)= & q_{f}(t)+k_{1} C^{*}(t)+k_{2} \int_{0}^{t}\left(C^{*}(\tau)-C_{r e f}^{*}(\tau)\right) d \tau \\
& +k_{3} C_{r e f}^{*}(t)
\end{aligned}
$$

The equivalent elevator command $u_{e}(t)$ has to be allocated on the existing control surfaces (i.e., the stabilizer and 4 the elevators). The free tuning parameters employed in the $C^{*}$ control law are collected in the 10 -dimensional vector

$$
\theta:=\left[k_{q}, T_{1}^{q}, T_{2}^{q}, V_{m}, k_{1}, k_{2}, k_{3}, k_{c}, T_{1}^{c}, T_{2}^{c}\right]
$$

and their optimal setting is the goal of the controller tuning.

The following criteria, depending on the tuning parameter $\theta$ and nonmeasurable uncertain parameter $\rho:=\rho_{1}=$ $\left(w, X_{c g}, V_{c}, h\right)$, have been used assuming a step signal for the demanded load factor $N_{z_{c}}(t)$ :

- $c_{1}(\theta, \rho)=\int_{t_{0}}^{t_{f}}\left(N_{z}(\tau)-N_{z_{r e f}}(\tau)\right)^{2} d \tau$, representing the integral of the squared regulation error over a time interval of interest $\left[t_{0}, t_{f}\right]$ (to be minimized), where $N_{z_{\text {ref }}}(\tau)$ is a reference load factor, $t_{0}$ is the initial time and $t_{f}$ is the final time when the steady state is presumably achieved;

- $c_{2}(\theta, \rho)=\max _{t \in\left[t_{0}, t_{f}\right]}\left|\left(q(t)-q\left(t_{f}\right)\right) / q\left(t_{f}\right)\right|$, representing the overshoot of $q(t)$; constrained to $c_{2}(\theta, \rho)<0.3$;

- $c_{3}(\theta, \rho)$ representing the settling time for $N_{z}(t)$, constrained to $5 \leq c_{3}(\theta, \rho) \leq 6$;

- $c_{4}(\theta, \rho)=\left|\dot{q}\left(t_{f}\right)\right| ;$ constrained to $c_{4}(\theta, \rho)<0.01$.

The constraint on $c_{4}$ serves as a stability enforcing requirement, while the bounds on $c_{2}$ and $c_{3}$ are requirements formulated in the RECONFIGURE Benchmark Problem.

To setup and solve the underlying multi-objective optimisation problem DLRs proprietary software environment MOPS [Joos, 1999] has been used. Instead using all 214 models for controller tuning purposes, an iterative procedure has been used to arrive to a set of representative design cases which can serve for the controller tuning. Starting with a few different load or flight cases and arbitrary initial values for the tuning parameters, efficient local search based optimization algorithms (e.g., pattern search and SQP methods) have been employed for tuning. A full assessment of performance requirements has been performed for all available flight and load cases, to determine possible violations of the design requirements. If there existed cases with unsatisfactory behavior, these have been added to the current set of design cases and the optimization run has been repeated. After a few iterations, a set of nine design cases has been found which ensured satisfactory performance over all possible design cases. For the selected cases, the local optimization based tuning algorithm needed not more than 200 criteria evaluations (about 20 minutes on a usual desktop PC) to obtain satisfactory solutions fulfilling all constraints.

Typical $C^{*}$ responses illustrating the excellent robustness of the optimally tuned $C^{*}$ control law are presented in Fig. 8 for the selected 9 design cases defined by the corresponding values of the uncertain parameters $\rho_{1}$. The requirement for the overshoot in $q$ is fully fulfilled, as illustrated in Fig. 9 for the same cases. The $N_{z}$ responses for a $N_{z_{c}}$ step, shown in Fig. 10, exhibit a certain scattering of the settling values, and contrast with the better performance achieved by the gain scheduling based normal law. 
This is however an expected performance degradation due to using a non-scheduled control law with only constant parameters.

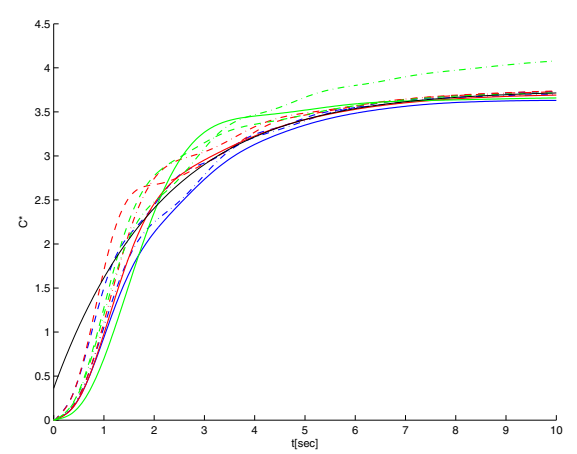

Fig. 8. $C^{*}$ responses for the 9 design cases.

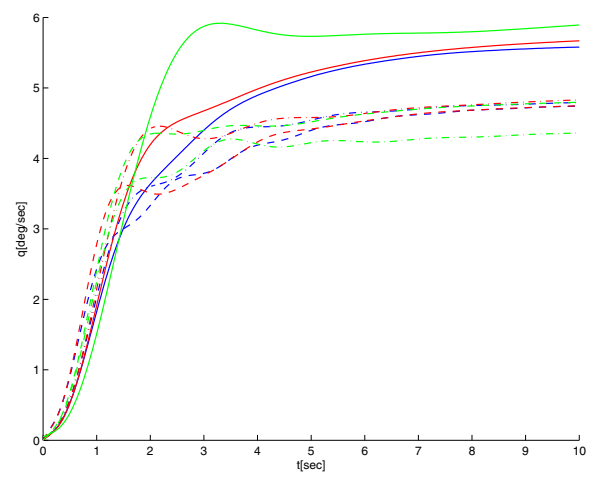

Fig. 9. Pitch rate responses for the 9 design cases.

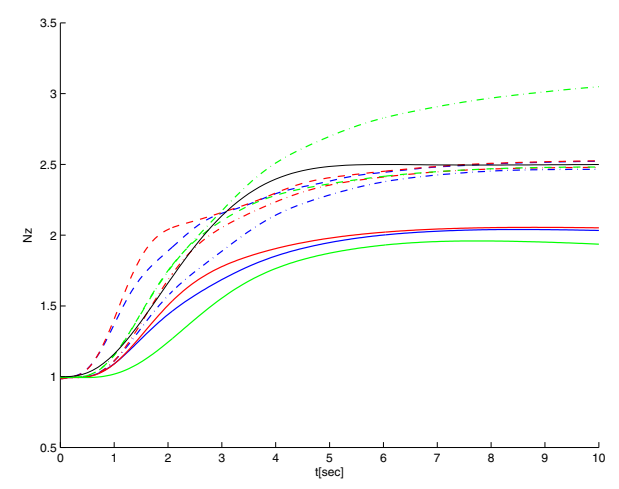

Fig. 10. $N_{z}$ responses for the 9 design cases.

\section{CONCLUSION}

We presented results for the design of the components of a proposed reconfigurable longitudinal flight control system which ensures satisfactory control performance even in the case of partial or total loss of air data sensory information. Linear multiple models based synthesis frameworks have been used for setting up both the underlying FDD systems as well as of a robust backup longitudinal control law. Additional increases in the FDD performce (e.g., lower detection thresholds) can be expected by employing better LPV-approximations (e.g., based on optimal fitting) for the residual generators. Although the results regarding the tuning of a robust backup longitudinal controller are still preliminary, they clearly demonstrate the possibility of using of a constant robust backup controller, without any gain scheduling. However, better performance can be expected by adding gain scheduling with respect to weight and/or center of gravity position, or even altitude or Mach number. A further refining of the control law, could be to perform the parameter tuning using the nonlinear aircraft dynamics model (instead of multiple linearized models), with built-in robustness guaranties by handling constraints via global worst-case search based techniques. Further work will also address the assessment of the overall FDDFTC performance using a full scale closed-loop nonlinear simulation model of the aircraft.

\section{REFERENCES}

P. Apkarian and D. Noll. Nonsmooth optimization for multidisk $\mathcal{H}_{\infty}$-synthesis. European Journal of Control, 12:229-244, 2006.

P. Goupil. AIRBUS state of the art and practices on FDI and FTC in flight control system. Control Engineering Practice, 19:524539, 2011.

G. Hardier, C. Seren, P. Ezerzere, and G. Puyou. Aerodynamic model inversion for virtual sensing of longitudinal flight parameters. In Proc. of 2nd Conference on Control and Fault-Tolerant Systems (SysTol'13), pages 25-30, 2013.

H.-D. Joos. A methodology for multi-objective design assessment and flight control synthesis tuning. Aerospace Science and Technology, 3:161-176, 1999.

H.-D. Joos, J. Bals, G. Looye, K. Schnepper, and A. Varga. A multi-objective optimisation-based software environment for control systems design. In Proc. of CACSD'2002, Glasgow, UK, 2002.

D. Ossmann and A. Varga. Optimization-based tuning of LPV fault detection filters for civil transport aircraft. In Proc. of 4th European Conference for Aerospace Sciences, St. Petersburg, Russia, 2011.

S. Oudin, G. Ferreres, G. Puyou, and P. Mouyon. Adaptive LFT control of a transport aircraft on the lateral axis. In Proc. of the IEEE Multi-Conference on Systems and Control (CCA Part), Dubrovnik, Croatia, pages 15341540, 2012.

G. Puyou and P. Ezerzere. Tolerance of aircraft longitudinal control to the loss of scheduling information: toward a performance oriented approach. In Proc. of the 7th IFAC Symposium on Robust Control Design (ROCOND'12), Aalborg, Denmark, pages 393-399, 2012.

C. Seren, G. Hardier, P. Ezerzere, and G. Puyou. Adaptive extended Kalman filtering for virtual sensing of longitudinal flight parameters. In Proc. of 2nd Conference on Control and Fault-Tolerant Systems (SysTol'13), pages 25-30, 2013.

A. Varga. On parametric solution of fault detection problems. In Proc. IFAC 2011 World Congress, Milano, Italy, pages 6697-6702, 2011a.

A. Varga. Synthesis of robust gain scheduling based fault detection filters for a class of parameter uncertain nonlinear systems. In Proc. of 19th Mediterranean Conference on Control and Automation, Corfu, Greece, pages 467-472, 2011b. 